\title{
Nonlinear dispersive scale Alfvén waves in magnetosphere-ionosphere coupling: Physical processes and simulation results
}

\author{
ZHAO MingXian ${ }^{1,2,3}$ \& LU JianYong ${ }^{3 *}$ \\ ${ }^{1}$ Chinese Academy of Meteorological Sciences, Beijing 100081, China; \\ ${ }^{2}$ Graduate University of Chinese Academy of Sciences, Beijing 100049, China; \\ ${ }^{3}$ National Center for Space Weather, China Meteorology Administration, Beijing 100081, China
}

Received September 4, 2011; accepted October 25, 2011; published online February 26, 2012

\begin{abstract}
Dispersive Alfvén waves (DAWs) have been demonstrated to play a significant role in auroral generation of the magnetosphereionosphere coupling system. Starting from a two fluid reduced MHD model, we summarize the frequency, temporal and spatial characteristics of magnetospheric DAWs. Then, the nonlinear kinetic and inertial scale Alfvén waves are studied, and we review some theoretical aspects and simulation results of dispersive Alfvén waves in Earth's magnetosphere. It is shown that dispersive standing Alfvén waves can generate the field-aligned currents which transport energy into the auroral ionosphere, where it is dissipated by Joule heating and energy lost due to electron precipitation. The Joule dissipation can heat the ionospheric electron and produce changes in the ionospheric Pedersen conductivity. As a feedback, the conducting ionosphere can also strongly affect the magnetospheric currents. The ponderomotive force can cause the plasma to move along the field line, and generate ionospheric density cavity. The nonlinear structuring can lead to a dispersive scale to accelerate auroral particle, and the Alfvén waves can be trapped within the density cavity. Finally, we show the nonlinear decay of dispersive Alfvén waves related to two anti-propagating electron fluxes observed in the auroral zone.
\end{abstract}

dispersive Alfvén wave, magnetosphere ionosphere coupling, auroral zone

Citation: Zhao M X, Lu J Y. Nonlinear dispersive scale Alfvén waves in magnetosphere-ionosphere coupling: Physical processes and simulation results. Chin Sci Bull, 2012, 57: 1384-1392, doi: 10.1007/s11434-011-4905-1

Alfvén waves in magnetosphere are to some extent an intimate part of dynamic auroral at small ionospheric scales, where the relevant scale-size ranges from the order of an electron skin depth to over $100 \mathrm{~km}$ [1-5]. Dispersive magnetohydrodynamic (MHD) waves with short-wavelength effect can transform energy from waves into particles [6]. Magnetospheric ULF Alfvén waves at short perpendicular scale are vital to the acceleration of auroral particles [7-9], and can be associated with all discrete auroral $[4,10]$. Chaston et al. [10] have shown that energy is transfered from magnetospheric ULF waves into auroral plasma through field-aligned electron acceleration, transverse ion acceleration and Joule heating, and the inclusion of nonlinear and/or nonlocal kinetic effects is required to describe these waves.

The auroral zone ionosphere is coupled to the magneto-

*Corresponding author (email: lujy@cma.gov.cn) sphere through field aligned currents (FACs) and energetic particle precipitation. Electron precipitation can be efficiently driven by quasi-linear wave-particle interaction [11-14], and the flux of energetic electrons can be well modeled using kinetic theory [15]. These FACs are carried by magnetospheric shear Alfvén waves (SAWs), and closed by field-crossing ionospheric currents [16]. Assuming that ionosphere is a thin conducting layer, it is reasonable to use height-integrated currents and conductivities to describe the closure of FACs and ionospheric currents. The current continuity equation is [17]

$$
j_{\|}=\nabla_{\perp} \cdot\left(\Sigma_{P} \boldsymbol{E}_{\perp}-\Sigma_{H} \boldsymbol{E}_{\perp} \times \hat{\boldsymbol{b}}\right),
$$

in which $\Sigma_{P}$ and $\Sigma_{H}$ are the ionospheric height-integrated Pedersen and Hall conductivities respectively. The subscripts $\perp$ and $\|$ denote vector components in the directions perpendicular or parallel to the magnetic field respectively.

There are two main theories which are widely used to 
interpret discrete auroral: the field line resonance (FLR) mechanism in which magnetosphere driven by external drivers (e.g. solar wind) plays the most important role [18], and the ionospheric feedback instability (IFI) mechanism in which magnetospheric FACs are strongly affected by the conducting ionosphere [19-22].

FLRs are standing SAWs that formed on closed geomagnetic filed lines in magnetosphere [23]. When driven by external forces, such as solar wind, the compressional waves propagate onto magnetic field lines in magnetosphere, on the condition that their frequencies match the local standing wave eigenfrequencies, they will change into shear waves which travel along Earth's geomagnetic field to auroral zone [4]. The auroral zone supports low frequency (1-4 mHz) standing shear Alfvén waves. Many researches [24, 25] have shown that FAC and certain other features of auroral arcs can be explained by FLRs. For example, electric fields, auroral electrons acceleration, and large scale vortex structures associated with sub-storms.

While many auroral arcs' characters, especially the periodical enhancement of auroral and the associated density cavities, can be successfully explained by FLRs $[16,25]$, the long oscillation period for FLRs does not match well to the observation data. Instead, IFI develops much faster to the typical scale of auroral arcs $[19,22,26]$. On the condition that there exists a large-scale background driving perpendicular electric field, a density/conductivity perturbation introduces a smallscale polarized electric field, whose direction is opposed to the original convection electric field. Because of current continuity requirement, the polarized electric field in ionosphere produces a FAC in magnetosphere which is carried by an upward propagating Alfvén wave. If the Alfvén wave reflected back from the anti-hemisphere or the magnetospheric equator has the synchronous phase with the density/conductivity enhancement, the perturbation in ionosphere as well as FAC and SAW in magnetosphere will be amplified, i.e. a ionospheric feedback instability is set up [19,27,28].

The dispersive processes become important when the perpendicular wavelength of SAW is in the order of the ion gyroradius $\rho_{s}$ [29], the ion thermal gyroradius $\rho_{i}$ [7] or the electron inertial length $\lambda_{e}$ [30]. The dispersive small-scale Alfvén wave is called the inertial Alfvén wave (IAW) in a medium where the electron thermal velocity $v_{t e}=\left(2 T_{e} / m_{e}\right)^{1 / 2}$ is less than Alfvén velocity $v_{A}$. In this case, the parallel electric field is supported by the electron inertia. Kinetic Alfvén wave (KAW) is wave in a medium where $v_{t e}>v_{A}$, and the parallel electric force is balanced by the parallel electron pressure gradient. Dispersive Alfvén wave (DAW) means both of IAW and KAW. The IAW appears when the plasma beta is low $\beta<m_{e} / m_{i}$, and KAW arises when $m_{e} / m_{i}<\beta<1$.

The nonlinear wave-wave interaction process and the excitation mechanisms of DAWs have been deeply studied [31-33]. The DAWs can be excited by turbulent, parametric instability, and modulational instability. Parametric instability of DAW has been investigated by many studies $[7,34]$.
One DAW can decay to another DAW and one ion acoustic wave due to parametric decay [35]. The DAWs can also be excited by other modes, such as whistler wave, the lower hybrid wave, the high-frequency radio wave, the ion cyclotron wave, the ion Bernstein wave, the ordinary electromagnetic wave, the Langmuir wave, and the MHD waves [34]. The DAW can also be decayed to themselves $[36,37]$.

\section{Magnetospheric FLRs with fixed iono- spheric boundary}

To investigate the characteristics of SAWs, Lu et al. [25] presented the full reduced MHD equations based on the work of Frycz et al. [38]:

$$
\begin{gathered}
\nabla \cdot\left[\frac{\rho \mu_{0}}{B_{0}^{2}}\left(1+\frac{3}{4} \rho_{i}^{2} \nabla_{\perp}^{2}\right) \frac{\mathrm{d}}{\mathrm{d} t} \nabla_{\perp} \phi\right]+\nabla \cdot\left(\boldsymbol{b} \nabla_{\perp}^{2} A\right)=\nabla_{\perp} \cdot\left(\frac{\mu_{0}}{B_{0}} \boldsymbol{b} \times \nabla_{\perp} P\right) \\
\frac{\partial A}{\partial t}+\boldsymbol{b} \cdot \nabla \phi=\lambda_{e}^{2} \frac{\partial}{\partial t} \nabla_{\perp}^{2} A+\frac{1}{e n_{e}} \boldsymbol{b} \cdot \nabla P_{e} \\
\rho \frac{\mathrm{d} V_{\|}}{\mathrm{d} t}=-\boldsymbol{b} \cdot \nabla P+\left(1+\frac{3}{4} \rho_{i}^{2} \nabla_{\perp}^{2}\right) \frac{\rho}{B_{0}^{2}} \nabla_{\perp} A \cdot \frac{\mathrm{d}}{\mathrm{d} t} \nabla_{\perp} \phi \\
\quad+\frac{1}{B_{0}} \boldsymbol{b} \cdot\left(\nabla_{\perp} A \times \nabla_{\perp} P\right) \\
\frac{1}{\rho} \frac{\mathrm{d} \rho}{\mathrm{d} t}=\frac{1}{B_{0}} \frac{\partial}{\partial t} \delta B_{\|}-\nabla \cdot\left(\boldsymbol{b} V_{\|}\right) \\
\nabla_{\perp} \delta B_{\|}=-\frac{\mu_{0}}{B_{0}} \nabla_{\perp} P-\frac{\mu_{0} \rho}{B_{0}^{2}} \boldsymbol{b} \times \frac{\mathrm{d}}{\mathrm{d} t} \nabla_{\perp} \phi
\end{gathered}
$$

where $\rho$ is the plasma density, $B_{0}$ is the background unperturbed magnetic field, $e$ is the elementary charge, $P$ is the plasma pressure, $P_{e}$ is the electron pressure, $n_{e}$ is the electron number density, $\boldsymbol{b}$ is the unit vector along the magnetic field, $V_{\|}$is the ion fluid velocity along the magnetic field, and $\delta B_{\|}$is the compressional perturbation of the magnetic field. These equations simulate the interaction between SAWs and ion acoustic waves (eqs. (4) and (5)), and include the effects of nonlinear process (density and pressure perturbation) in eq. (2), finite ion gyroradius, electron inertia, and electron thermal pressure in eq. (3). Eq. (6) is derived from Ampere's law.

The plane wave solutions to the linear reduced two-fluid MHD equations satisfy the local dispersion relation [4]:

$$
\omega^{2} \sim k_{\|}^{2} V_{A}^{2}\left(\frac{1+\frac{3}{4} k_{\perp}^{2} \rho_{i}^{2}+k_{\perp}^{2} \rho_{s}^{2}}{1+k_{\perp}^{2} \lambda_{e}^{2}}\right),
$$

where $k_{\|}$and $k_{\perp}$ are the parallel and perpendicular wave number respectively, $\lambda_{e}=c / \omega_{p e}$ is the electron inertial length, $\rho_{s}=C_{s} / \Omega_{i}$ is the ion acoustic gyroradius with $\Omega_{i}$ the ion gyro-frequency and $C_{s}$ the sound speed. This dispersion relation is valid for $k_{\perp}^{2} \lambda_{e}^{2}\left(\rho_{s}^{2}\right)<1$, and is appropriate for waves that have small parallel wavelength along the field line, e.g. traveling waves. 
The equations describing the amplitude evolution of the shear wave magnetic field are shown as [4]

$$
\begin{gathered}
\frac{\partial b}{\partial t}-i \omega_{0} \frac{\partial}{\partial x}\left(\delta \frac{\partial b}{\partial x}\right)=+i \Delta \omega b+\omega_{0} R \\
\delta=L^{2} R_{e}^{2} \int \mathrm{d} l\left(\frac{3}{4} \frac{\rho_{s}^{2}}{\omega_{0}^{2}} \frac{V_{A}^{2}}{h_{3}}\left(\partial_{l} S_{1}\right)^{2}+\frac{V_{T e}^{2}}{\omega_{0}^{2} h_{3}}\left(\partial_{l} S_{1}\right) \partial_{l}\left(S_{1} \lambda_{e}^{2}\right)-\frac{\lambda_{e}^{2}}{h_{3}} S_{1}^{2}\right) .
\end{gathered}
$$

The term $\Delta \omega(x)=\omega_{0} x / l_{\omega}$ measures the increase or decrease in the eigenfrequency of SAWs, and the change rate of frequency across field line depends on $l_{\omega}$, the local gradient in Alfvén speed. $\delta(x)$, the so-called dispersion parameter, represents the weighted-average of wave dispersion along a field line, it is positive when thermal effects dominant and negative when electron inertial effects dominant. In a Cartesian geometry, eq. (9) will be simplified to $\delta=\delta_{i}+\delta_{e}=$ $\frac{3}{4} \rho_{i}^{2}-\lambda_{e}^{2}\left(1-V_{T e}^{2} / V_{A}^{2}\right)[24]$.

In the absence of dispersion, the phase mixing time is dependent on the density and temperature of the background plasma. In the case of standing waves, accounting for wave dispersion, and ignoring the radial dependence of dispersion parameter $\delta$, the dispersive saturation timescale, width, and amplitude are estimated as follows [39-41]:

$$
\omega_{0} t_{\mathrm{dis}}=2\left(\frac{l_{\omega}^{2}}{|\delta|}\right)^{1 / 3}, l_{\mathrm{dis}}=\left(|\delta| l_{\omega}\right)^{1 / 3}, b_{\mathrm{dis}}=R\left(\frac{l_{\omega}^{2}}{|\delta|}\right)^{1 / 3} .
$$

(a) Linear evolution

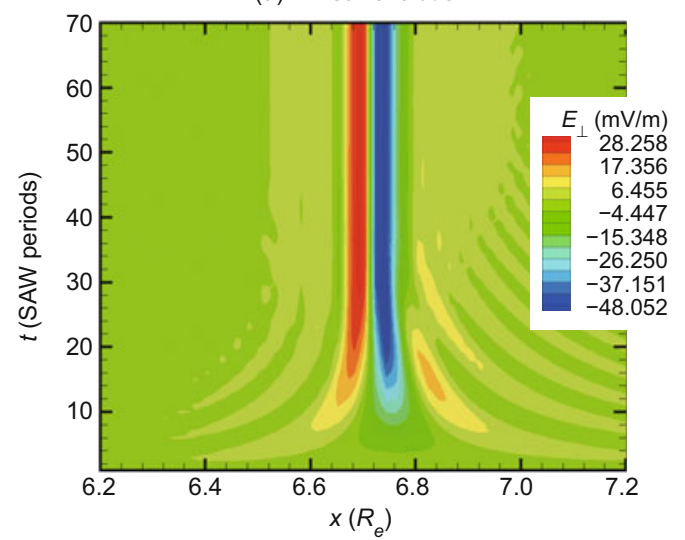

The saturation time and width are independent of the amplitude of the excitation source. In regions where dispersion is large, the width will be large and the wave amplitude will be small. In contrast, at the point where dispersion is small, very short scale is generated (i.e. $\delta$ is small). Phase mixing is also affected by gradients in wave dispersion across geomagnetic field lines, which act to defocus SAWs, causing wave energy to propagate Earthward (in the equatorial plane) onto field lines where dispersion is small [42].

The phase-mixing timescale and width are also affected by the gradient in the Alfvén speed profile perpendicular to geomagnetic field lines. The including of density variation is important in the formation of small scale (comparable to $\lambda_{e}$ and $\rho_{s}$ ) waves and the density cavities with scale of few $\mathrm{km}$ at 1500-2000 km altitude [43,44]. Lu et al. [23, 25] solved the eqs. (2)-(6), investigated the dynamic evolution of nonlinear FLR.

The dynamic evolution of electric field component perpendicular to the magnetic field at the equatorial plane in the linear and nonlinear cases in dipole geomagnetic field are shown in Figure 1(a) and (b), and the case of nonlinear evolution in stretched geomagnetic field is shown in Figure 1(c). In the linear case in which the ponderomotive forces are neglected, the imposed driver causes the wave energy to narrow and grow strong, finally lead to a static standing Alfvén wave. The dispersive processes do not take apparent effect on the

(b) Nonlinear evolution

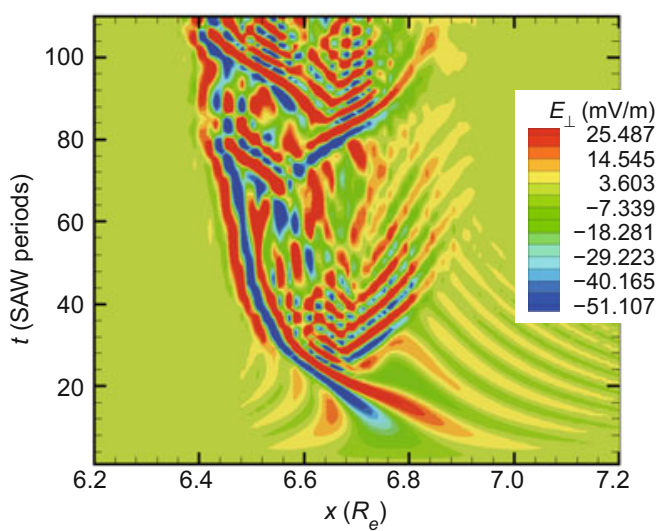

(c) Nonlinear evolution

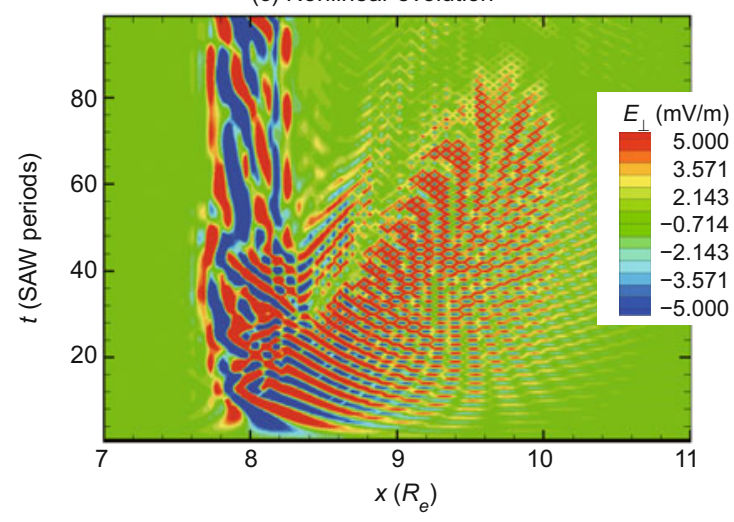

Figure 1 Dynamic evolution of electric field component perpendicular to the magnetic field $E_{\perp}$ at the equatorial plane in the (a) linear case in dipole geomagnetic field, (b) nonlinear case in dipole geomagnetic field, and (c) nonlinear case in stretched geomagnetic field [23,25]. 
propagation of wave energy as dispersive scales are too small compared to the width of the standing wave. In the nonlinear case illustrated in Figure 1(b), the initial temporal evolution is similar to the linear case as nonlinear and dispersive effects are not important yet. As the developing of FLRs, the ponderomotive force drives density perturbations and produces a nonlinear steepening of the perpendicular profile of the Alfvén velocity. Correspondingly, the plasma eigenfrequency changes, the resonance position move Earthward, and the wave structure is broader and more complicate. Not all dispersive effects drive the resonance in the same direction, while ponderomotive force and electron inertia attempt to move the resonance Earthward, thermal effects work in the opposite direction. However, the perpendicular electric field is too small compared to the observation data in the box model which are not shown here, and too large in the dipolar model as shown in Figure 1(a) and (b). A more realistic stretched magnetic field configuration is implied to explain observations above the auroral ionosphere and the result is in Figure 1(c). Figure 1(c) shows the time-dependent nonlinear spatial structuring of the dispersive FLR in the equatorial plane. In this case, the dispersive effects are much stronger and $E_{\perp}$ is significantly smaller than in the dipolar case. The stretching of field line also brings the FLR frequencies to the range of observation.

In dipolar field, the density perturbations driven by ponderomotive force move the plasma along magnetic field from high latitude to equator, and form the so called ionospheric density cavity and density bump around equator in which the FLR is trapped inside. But in the stretched field, electron thermal pressure result in anti-Earthward density perturbation propagation which vanish eventually.

As the density perturbation narrowed with a small region,
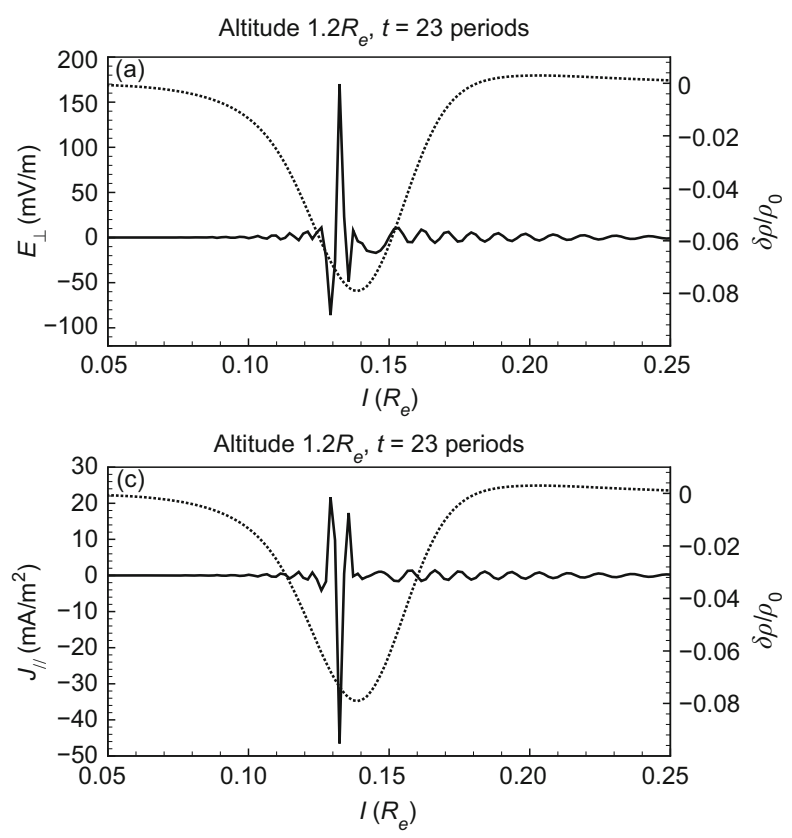

which is observed as density cavity by satellite, the resonance frequency is reduced and the dispersion is enhanced. As shown in Figure 2, the density cavity eventually trap the shear Alfvén waves.

\section{Ionospheric electron heating}

In magnetosphere-ionosphere (M-I) coupling system, FACs carried by SAWs are closed through Pedersen and Hall currents in the ionosphere. In the rest of this article, we shall neglect the Hall current for simplicity. The ionospheric Pedersen conductivity, which is associated with the electron density, is important in feedback effects to magnetospheric SAWs. The electron production in the ionosphere is derived from photoionization and/or electron impact ionization and from chemistry, while electron losses are mainly due to ionneutral reactions and electron-ion recombination [45].

By ignoring the chemical production/losses, the electron density continuity equation will be $[16,20,28,45]$

$$
\frac{\partial n_{e}}{\partial t}=v_{\mathrm{ioniz}} n_{e}-R\left(n_{e}^{2}-n_{e 0}^{2}\right)-\frac{j_{\|}}{e h}+\gamma_{\mathrm{hot}},
$$

where $n_{e}$ is the electron density and $n_{e 0}$ is background of $n_{e}$, $v_{\text {ioniz }}=0.1 v_{e} \exp \left(-\varphi / T_{e}\right)$ is the ionization rate in which $v_{e}$ is the collision frequency of electron with neutrals, $R$ is the constant of recombination, and the last two terms are external sources from FACs and auroral electron precipitation respectively.

The energy balance equations in a reference moving with neutrals are $[45,46]$

$$
\frac{3}{2} n_{e, i} \frac{\partial T_{e, i}}{\partial t}+n_{e, i} T_{e, i} \nabla \cdot \boldsymbol{u}_{e, i}+\nabla \cdot \boldsymbol{q}_{e, i}=Q_{e, i}-L_{e, i}
$$
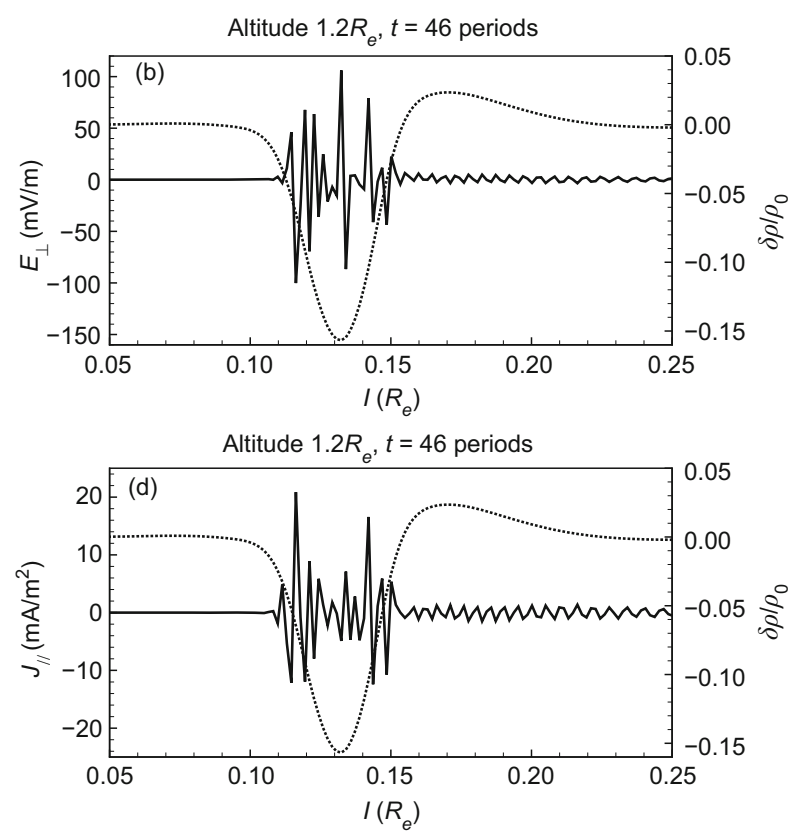

Figure 2 Radial dependence of $E_{\perp}$ and $j_{\|}$at altitude $1.2 R_{E}$ for $t=23$ and 46 periods, respectively. The dotted line is the relative density perturbation [25]. 


$$
\frac{3}{2} n_{n} \frac{\partial T_{n}}{\partial t}+\nabla \cdot \boldsymbol{q}_{n}=L_{e}+L_{i}
$$

In eq. (12), the second term on the left side stands for heat convection, and the third is conduction term. $Q_{e, i}$ is the energy released in the corresponding charge, and $L_{e, i}$ is the energy loss due to collision with neutrals.

In the stationary approximation, neglecting diffusion/ convection losses, one can get the critical Pedersen current $[45,46]$ :

$$
j_{c}=\sqrt{\frac{v_{i} / \Omega_{i}}{v_{e} / \Omega_{e}} \frac{\sigma_{P 0} n_{e 0} T_{*}}{1+v_{i}^{2} / \Omega_{i}^{2}} \sum\left(\frac{3 m_{e} v_{e n} k}{m_{n}}+L_{e}\left(T_{e}=T_{*}\right)\right)},
$$

where $T_{*}$ corresponds to the balance between collision ionization and recombination. In the linear regime when ionospheric current $j_{\perp}<j_{c}, T_{e}$ increases to $T_{*}$, the change of electron density is negligible, and ionization is not important. In the nonlinear regime, $T_{e}$ is saturated to $T_{*}$, ionization becomes important, $n_{e}$ increases and so is the Pedersen conductivity $\sigma_{P}$.

Figure 3 shows the critical currents required for electron heating to be effective. The critical current increases with initial conductivity. When the current exceeds the critical current, the nonlinear heating regime comes into play, and this regime depends on the ionospheric current, not the initial conductivity.

It is shown that ionospheric electrons are heated mainly by Joule dissipation from SAWs, and are cooling due to ionization losses and collisions with neutrals. The 2D simulation results of Lu et al. [45] also shown that ionospheric conductivity strongly feedback magnetospheric waves.

\section{Interactive magnetosphere-ionosphere coupling}

Ionosphere is usually treated as boundary layer in magnetosphere-ionosphere coupling model, and the FACs carried by SAWs are strongly affected by conducting ionosphere. On the other hand, the conductivities along the magnetospheric field lines which supporting the SAWs play an important role in the evolution of FLRs. However, early M-I models [47,48]

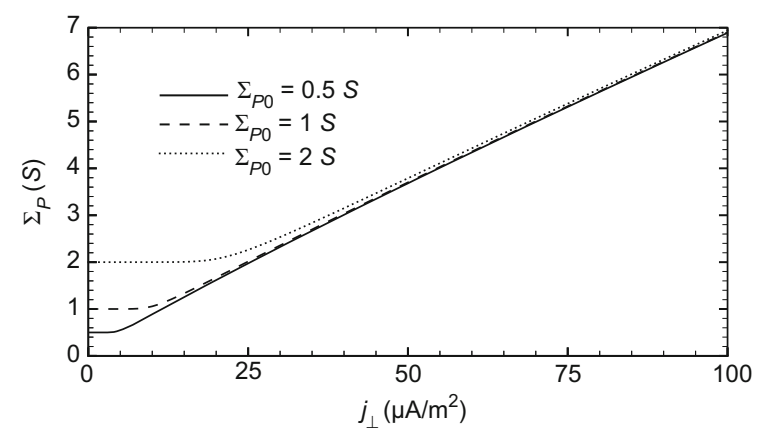

Figure 3 Pedersen conductivity as a function of ionospheric current for $\Sigma_{P 0}=0.5 S$ (solid), $1 S$ (dashed), and $2 S$ (dotted) [46]. rarely care about dynamic interaction of magnetosphere and ionosphere. They either focused on the ionospheric response to a fixed Alfvén wave input, or used a fixed ionospheric boundary.

Prakash et al. [49] investigated the interaction between ionospheric feedback, nonlinear, and dispersive effects based on a envelope mode, finding that hundreds $\mathrm{eV}$ electrons can produce strong enhancements of FACs. However, the electron precipitation introduced Pedersen conductivity is simply treated by an empirical formula, and the precipitation energy is an independent parameter. Further more, this mode did not include the effects of field-aligned potential drops and the magnetic mirror force. Lu et al. [45, 46] investigated the feedback on the linear FLR amplitude caused by heated electrons from Joule dissipation for large current system. These works shown that it is important to treat the wave and ionospheric conductivities properly in M-I coupling involving shear Alfvén waves.

Lu et al. [16,28] extended the work of Prakash et al. [49] to full MHD caculation. The full wave nonlinearities, including its coupling with compressional modes, are accounted for. The magnetospheric FACs are coupled to ionospheric Pedersen currents through eqs. (1) and (11).

To caculate the ionospheric $E$ region electron precipitated ionization rate $\gamma_{\mathrm{hot}}$, FACs are related to characteristic energy (or average energy) and energy flux of particle precipitation through Knight-relationship. Firstly, the initial energy $\varepsilon_{0}$ and particle flux $\Psi_{0}$ are given as $[16,50,51]$

$$
\varepsilon_{0}=\alpha C_{s}^{2}, \quad \Psi_{0}=\beta \rho \varepsilon_{0}^{1 / 2},
$$

where $\alpha$ and $\beta$ are constant parameters which map the magnetospheric plasma thermal flux and energy to the ionosphere. Secondly, field-aligned potential drop $\varepsilon_{\|}$is given by $[16,52,53]$

$$
\varepsilon_{\|}=C j_{\|} \varepsilon_{0}^{1 / 2} / \rho
$$

where $C$ is an adjustable scaling factor for the potential drop and includes an "effective resistivity" to FACs. Then, the particle flux $\Psi$ and average energy $\varepsilon$ are obtained as [16]

$$
\begin{gathered}
\Psi=\Psi_{0}\left(8-7 \exp \left[-\varepsilon_{\|} /\left(7 \varepsilon_{0}\right)\right]\right) \quad \varepsilon_{\|}>0, \\
\Psi=\Psi_{0} \exp \left[\varepsilon_{\|} / \varepsilon_{0}\right] \quad \varepsilon_{\|}<0, \\
\varepsilon=\varepsilon_{0}+\varepsilon_{\|} .
\end{gathered}
$$

Finally, $\Psi$ and $\varepsilon$ are input into a thermosphere-ionosphere model to obtain the ionization rate. The ionospheric ionization model used by Lu et al. [16,28] is GLOW, which is a physics model and was developed at NCAR/HAO.

Two types of shear Alfvén wave sources to produce the ionospheric conductivity enhancements are studied $[16,28]$ : magnetospheric FLRs and ionospheric feedback instability. It is shown that the auroral electron precipitation-induced Pedersen conductivity enhancement can lead to strong feedback effects on magnetospheric FLR wave amplitudes and density perturbation. 
As shown in Figure 4, the ionospheric feedback due to the auroral electron precipitation bring the amplitude of fieldaligned current $j_{\|}$to 3 from $0.5 \mu \mathrm{A} / \mathrm{m}^{2}$ in dipolar case, and to 7 from $2 \mu \mathrm{A} / \mathrm{m}^{2}$ in stretched case. It means that when auroral electron precipitation is included, the Pedersen conductivity increases, and SAW dissipation is reduced. The ionospheric feedback depends on the competition between precipitation energy and wave damping. Although the higher wave damping may reduce FAC, the stretching of magnetic field can bring about a larger parallel current and magnetic perturbation. The precipitation energies required to initiate the feedback effect in a stretched case are lower than in the case of a dipolar field.

Nonlinear effects can produce strongly localized FLRs and density perturbation is strongly enhanced when auroral electron precipitation is included. Figure 5 shows the relative density perturbation. Along the resonant field line, the plasma moves from high latitudes to the equator, which is similar to the reduced MHD simulation result $[23,25]$, resulting in density cavity in high latitudes and density bump
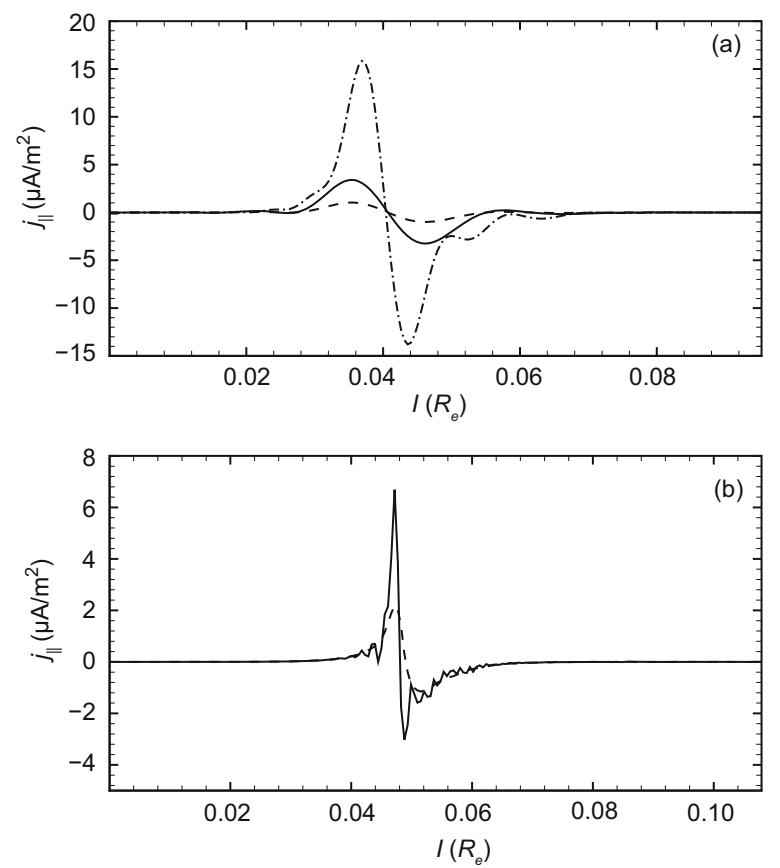

Figure 4 (a) Comparison of parallel current $j_{\|}$at ionospheric boundary between $\Sigma_{P}=\infty$ (dash dotted), $\Sigma_{P}=1 S$ (dashed) and the interactive model with $\Sigma_{P 0}=1 S$ as initial condition (solid) in dipolar field. (b) Comparison of $j_{\|}$at ionospheric boundary between fixed $\Sigma_{P}=1 S$ (dashed) and the interactive model (solid) in stretched field (combined from Figures 1 and 4 in Lu et al. [16]).

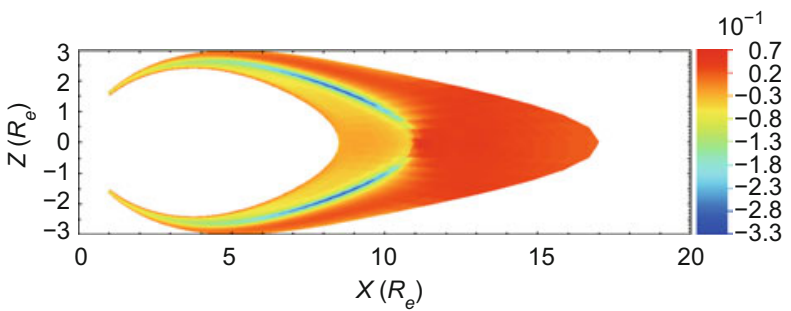

Figure 5 Relative density perturbation of Topo-GLOW coupling model at $t=12$ periods [16]. at the equator. However, there exist significant movements of plasma across stretched field lines in full MHD while the density perturbation is mainly along the field lines in reduced MHD computation. This implies that around the equator, where plasma $\beta$ is high, the reduced MHD breaks down and new behavior can occur due to high plasma pressure effect.

The feedback instability can be triggered by a very smallscale, small amplitude density perturbation (only 1\%), and the small-scale wave structures observed by satellites can be attributed to the waves triggered by feedback instability. The auroral electron precipitation can strongly enhance magnetospheric wave amplitudes and density perturbation, and both the ionospheric density perturbation and magnetospheric waves are influenced by the effects that they produce. Precipitating energy and energy flux significantly affect the growing speed of the feedback instability. Higher precipitation energy and energy flux leads to faster instability growths. However, for lower amplitude FACs, auroral precipitating electrons have no significant effect in feeding back the magnetospheric waves.

The plasma movements in the feedback instability case are very different from that associated with FLRs. The compare of these two cases are shown in Figure 6. In an instability, across the field lines at the equatorial plane, there is plasma moving earthward and anti-earthward; while in FLR, the plasma mainly moves along the field line from high to
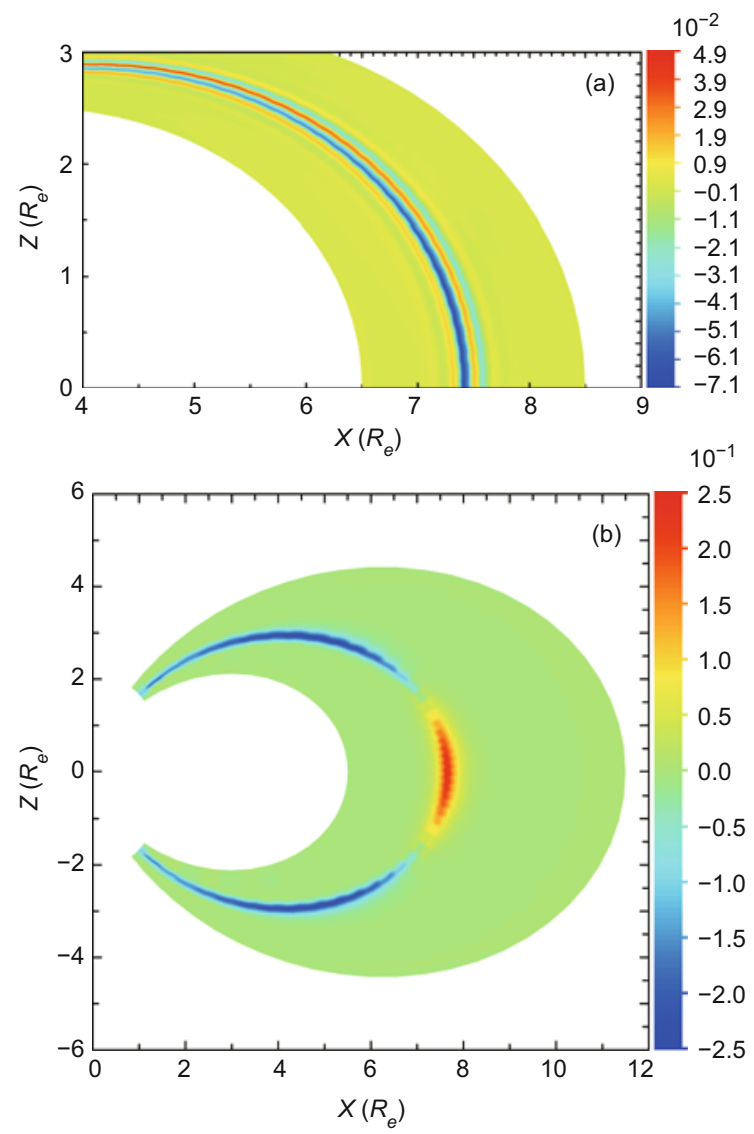

Figure 6 Spatial distribution of the relative density perturbation for (a) the instability case and (b) FLR case [28]. 
low latitude (at least for low $\beta$ plasma). As a result, density redistributions are different between FLRs and the instabilities: In FLR, the plasma mainly moves along the field lines, especially on the resonance shell, resulting in a density bump at the equator and a cavity in the high latitudes close to the ionosphere; while in the feedback instabilities, density is distributed either as bump or cavity along the field line, and across the field lines, cavity and bump occur alternately. The cavity with very large scale and large amplitude across the field line is more possibly generated by FLRs and the higher frequency density fluctuation can be attributed to the feedback instability.

The simulated effects of seasonal conductivity asymmetry are shown in Figure 7, in which the initial winter (northern) ionospheric Pedersen conductivity is $1 S$ and that of summer (southern) is $3 S$. The perpendicular electric field $E_{\perp}$ shows strong asymmmetry between two hemisphere, which agrees well with observed conclusion of auroral arcs $[54,55]$.

\section{Nonlinear decay of dispersive Alfvén waves}

The parametric instability has been studied by many authors to investigate the excitation of DAWs [36,37]. However, previous studies only focused on the decay mechanism in which two decay DAWs are parallel propagation waves.

Zhao et al. [34] extend these work to more general plasma $\beta$ parameters, including the inertial region with $\beta<Q$ and high $\beta$ region with $\beta \sim 1$, even $\beta>1$. Two decay cases of DAW were discussed: the parallel decay case where two decay waves have the same direction, and the reverse decay case where two decay waves are reverse propagating. The restrict relation was obtained from the resonant condition and dispersion relation:

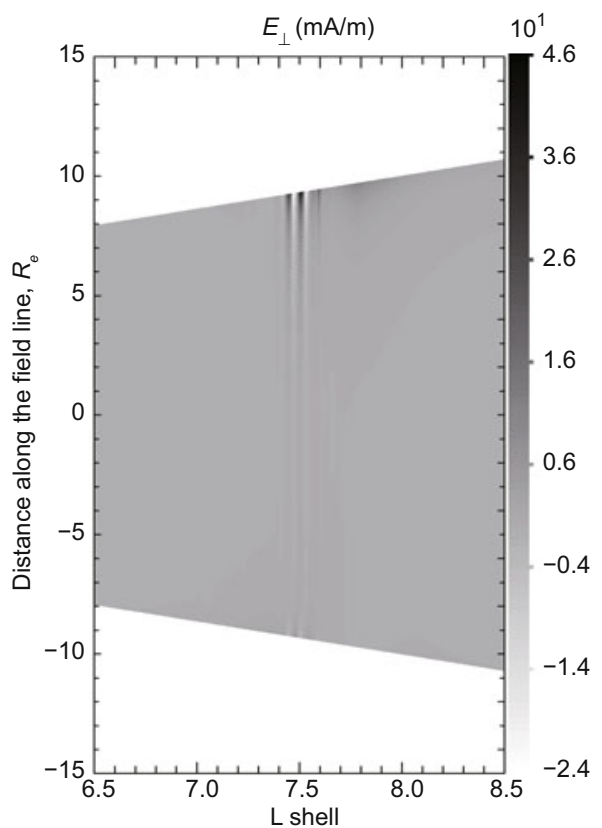

$$
k_{1 z}\left(s_{1} K_{0}-K_{1}\right)=k_{2 z}\left(K_{2}-s_{2} K_{0}\right) .
$$

If the growth rate of the decay wave is much smaller than its real frequency, the growth rate can be written as

$$
\begin{aligned}
& \gamma^{2}=-\frac{1}{16} \frac{\left|b_{0}\right|^{2}}{B_{0}^{2}} \frac{V_{A}^{2} K_{1} K_{2} \sin ^{2} \theta}{k_{0 \perp}^{2}}\left(1+\lambda_{e}^{2} k_{1 \perp}^{2}\right) \\
& \times\left(1+\lambda_{e}^{2} k_{2 \perp}^{2}\right)\left(1+\lambda_{e}^{2} k_{0 \perp}^{2}\right)^{2}\left(K_{1}-s_{1} K_{0}\right)\left(K_{2}-s_{2} K_{0}\right) \\
& \times\left(\frac{1}{K_{0}} \frac{k_{0 \perp}^{2}}{1+\lambda_{e}^{2} k_{0 \perp}^{2}}+\frac{s_{1}}{K_{1}} \frac{k_{1 \perp}^{2}}{1+\lambda_{e}^{2} k_{1 \perp}^{2}}+\frac{s_{2}}{K_{2}} \frac{k_{2 \perp}^{2}}{1+\lambda_{e}^{2} k_{2 \perp}^{2}}\right)^{2} .
\end{aligned}
$$

In the inertial region, where electron inertial effect is more important than finite ion gyro-radius effect, the reverse decay is stronger than the parallel decay, and the decay rate of the pump wave into the shorter-wavelength daughter waves is higher than that into the longer-wavelength daughter waves, implying that the decay process develops mainly towards to exciting small-scale waves. In the kinetic region and high $\beta$ region, the nonlinear growth rate decreases with $\beta$, but increases with the ion-electron temperature ratio $T_{i} / T_{e}$.

The results of DAWs decay have also been applied to auroral zone. Based on the background conditions used in Kletzing and Torbert [56], Kletzing et al. [57], Lysak and Lotko [58], and Chaston et al. [59], Zhao et al. [34] calculated the evolution of the plasma $\beta$ and the perpendicular wave number shown in Figure 8(a), and the change of the maximal growth rates shown in Figure 8(b). Approximately, the region with $r$ from $1 R_{E}$ to $5 R_{E}$ is the inertial region, and above $5 R_{E}$ is kinetic region. $\lambda_{e} k_{\perp}$ is small at low altitude and reach maximum value at $r \sim 2 R_{E}$, which is the typical auroral acceleration region. Figure 8(b) shows that there is a transform point at $r \sim 5 R_{E}$, and the reverse decay is always stronger than the parallel decay, especially in the inertial range. These results

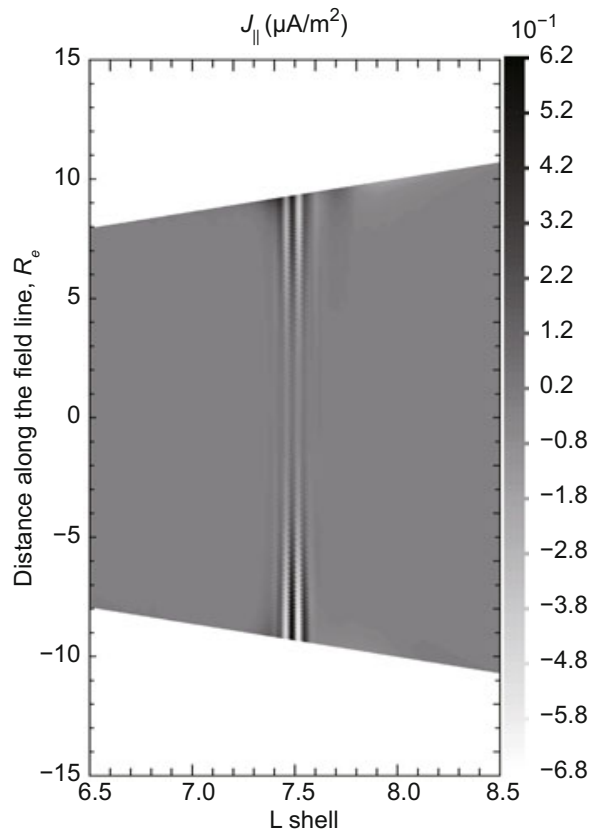

Figure 7 Spatial distributions of perpendicular electric field and parallel current density for seasonal effects of conductivity asymmetry [28]. 

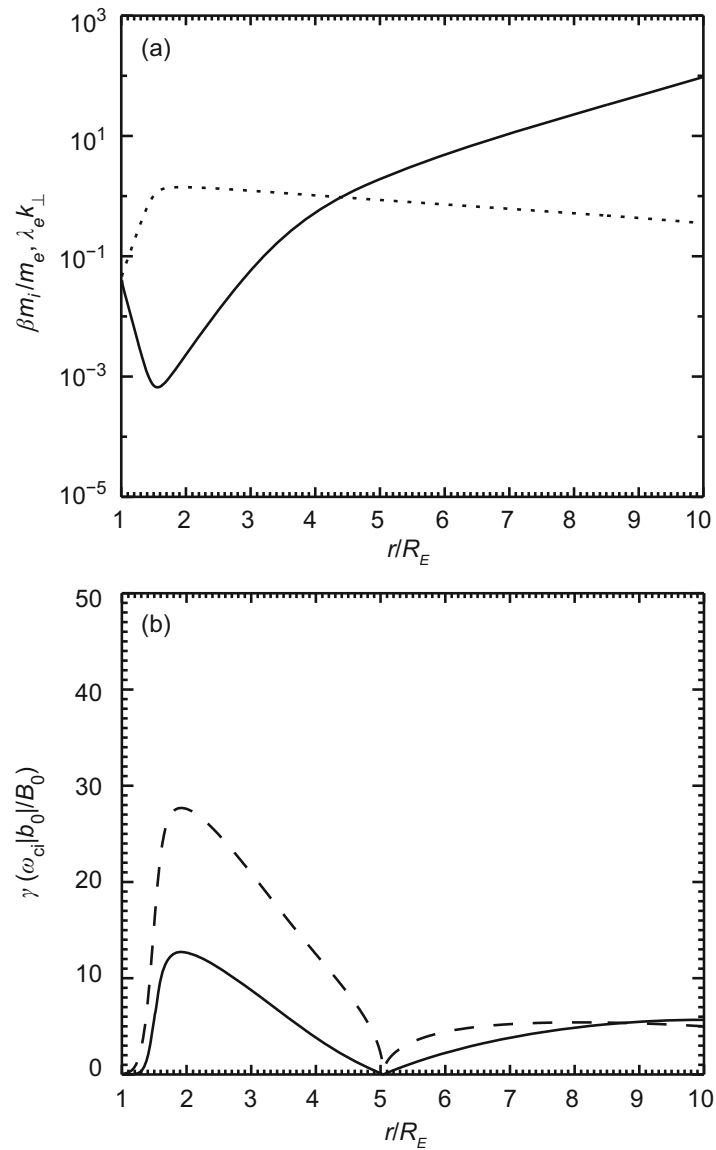

Figure 8 (a) The evolution of parameters $\beta m_{i} / m_{e}$ (solid) and $\lambda_{e} k_{\perp}$ (dotted) with the radial distance in the auroral zone. (b) The maximal growth rates change with the radial distance in parallel decay case (solid) and reverse decay (dashed) [34].

agree well with previous studies and observations [58-60].

It is also calculated [34] that the threshold amplitudes are very small and can be easily satisfied in the auroral zone, which means the parametric decay can easily occur in the electron acceleration range and may play an important role in accelerating electron there. This nonlinear decay mechanism can also be used to explain the excitation of reverse propagating electrons fluxes [61].

Zhao et al. [62] studied the nonlocal coupling between dispersive Alfvén waves and large-scale Alfvén waves (AWs). The results shown that in the inertial region, the decay occurs in the way of AW to DAW1 + DAW2, but in the kinetic region, the way is AW + DAW1 to DAW2. These two processes both can directly transfer the wave energy from the large-scale AWs to the small-scale DAWs.

\section{Summary}

In this article, we give a review on the basic theory of shear Alfvén waves at short perpendicular spatial scale. The frequency, temporal and spatial characteristics are summarized based on the envelope two-fluid reduced MHD equations. The nonlinear evolution processes based on the simulation results of two-fluid reduced MHD are listed and it is shown that different dispersive processes have different effects on the resonance waves.

We also summarize the studies of magnetosphere-ionosphere coupling simulation, especially the full MHD model that describes the dynamic interaction between magnetospheric waves, compressional modes, and auroral electron precipitations. The nonlinear electron heating by SAWs is calculated and the heated electrons by SAWs can cause ionization which change the Pedersen conductivity. Time dependent dispersion and density steepening lean to the acceleration of dispersive effects and to localization of the FLR. SAWs are trapped between turning points inside density perturbations. Ponderomotive density redistribution and field line stretching lead to reduction of eigen-frequencies. Two mechanisms (FLR and IFI) are shown, which are commonly used to explain the formation of FAC, auroral arcs, density cavity and bump. FLR performs well in explaining the very large scale and large amplitude density cavity, and IFI is more suitable to explain the higher frequency density fluctuation.

We finally discuss the nonlinear decay of the kinetic Alfvén waves. The parametric instability may play an important role in the forming of electron beams observed in auroral zone.

This work was supported by the National Natural Science Foundation of China (40874087 and 41031063), the China Meteorological Administration (GYHY201106011) and the China Public Science and Technology Research Funds Projects of Ocean (201005017).

1 Borovsky J E. Auroral arc thicknesses as predicted by various theories. J Geophys Res, 1993, 98: 6101-6138

2 Trondsen T S, Cogger L L, Samson J C. Asymmetric multiple auroral arcs and inertial Alfvén waves. Geophys Res Lett, 1997, 24: 2945-2948

3 Knudsen D J, Donovan E F, Cogger L L, et al. Width and structure of mesoscale optical arcs. Geophys Res Lett, 2001, 28: 705-708

4 Rankin R, Watt C E J, Kabin K, et al. Theoretical aspects of kinetic and inertial scale dispersive Alfvén waves in Earth's magnetosphere. Geophys Monogr, 2006, 169: 91-108

5 Zhang C M, Wei Y C, Yin $\mathrm{H} \mathrm{X}$, et al. The emission positions of $\mathrm{kHz}$ QPOs and Kerr spacetime influence. Sci China Phys Mech Astron, 2010, 53: 114-116

6 Chen L, Wu D J. Exact solutions of dispersion equation for MHD waves with short-wavelength modification. Chin Sci Bull, 2011, 56: 955-961

7 Hasegawa A. Particle acceleration by MHD surface wave and formation of aurora. J Geophys Res, 1976, 81: 5083-5090

8 Goertz C K. Kinetic Alfvén waves on auroral field lines. Planet Space Sci, 1984, 32: 1387-1392

9 Chaston C C, Carlson C W, Ergun R E, et al. Alfvén waves, density cavities and electron acceleration oberved from the FAST spacecraft. Physica Scripta, 2000, T84: 64-68

10 Chaston C C, Peticolas L M, Carlson C W, et al. Energy deposition by Alfvén waves into the dayside auroral oval: Cluster and FAST obervations. J Geophys Res, 2005, 110: A02211

11 Xiao F, Su Z, Zheng $\mathrm{H}$, et al. Modeling of outer radiation belt electrons by multidimensional diffusion process. J Geophys Res, 2009, 114 : A03201

12 Xiao F, Zong Q G, Chen L. Pitch-angle distribution evolution of energetic electrons in the inner radiation belt and slot region during the 2003 Halloween storm. J Geophys Res, 2009, 114: A01215 
13 Xiao F, Su Z, Zheng H, et al. Three-dimensional simulations of outer radiation belt electron dynamics including cross-diffusion terms. J Geophys Res, 2010, 115: A05216

14 Xiao F, Su Z, Chen L, et al. A parametric study on outer radiation belt electron evolution by superluminous R-X mode waves. J Geophys Res, 2010, 115: A10217

15 Li L, Feng Y Y. Energetic electron flux distribution model in the inner and middle magnetosphere. Sci China Tech Sci, 2011, 54: 441-446

$16 \mathrm{Lu} \mathrm{J} \mathrm{Y,} \mathrm{Rankin} \mathrm{R,} \mathrm{Marchand} \mathrm{R,} \mathrm{et} \mathrm{al.} \mathrm{Electrodynamics} \mathrm{of} \mathrm{magneto-}$ sphere-ionosphere coupling and fedback on magnetospheric field line resonances. J Geophys Res, 2007, 112: A10219

17 Paschmann G, Haaland S, Treumann R. Auroral plasma physics. Space Sci Rev, 2002, 103: 1-475

18 Lysak R L. Electrodynamic coupling of the magnetosphere and ionosphere. Space Sci Rev, 1990, 52: 33-87

19 Atkinson G. Auroral arcs: Result of the interaction of a dynamic mag netosphere with the ionosphere. J Geophys Res, 1970, 75: 4746-4755

20 Sato T. A theory of quiet auroral arcs. J Geophys Res, 1978, 83: 1042 1048

21 Miura A, Sato T. Numerical simulation of global formation of auroral arcs. J Geophys Res, 1980, 85: 73-91

22 Streltsov A, Lotko W, Milikh G M. Simulation of ULF field-aligned currents generated by HF heating of the ionosphere. J Geophys Res, 2005, 110: A04216

23 Lu J Y, Rankin R, Marchand R, et al. Nonlinear acceleration of dispersive effects in field line resonances. Geophys Res Lett, 2003, 30: 1540

24 Rankin R, Samson J C, Tikhonchuk V T, et al. Auroral density fluctuations on dispersive field line resonances. J Geophys Res, 1999, 104: 4399-4410

25 Lu J Y, Rankin R, Marchand R, et al. Finite element modelling of nonlinear dispersive field line resonances: Trapped shear Alfvén waves inside field-aligned density structures. J Geophys Res, 2003, 108: 1394

26 Lysak R L, Song Y. Energetics of the ionospheric feedback interaction. J Geophys Res, 2003, 107: 1160

27 Watanabe T H. Feedback instability in the magnetosphere-ionosphere coupling system: Revisited. Phys Plasmas, 2010, 17: 022904

28 Lu J Y, Wang W, Rankin R, et al. Electromagnetic waves generated by ionospheric feedback instability. J Geophys Res, 2008, 113: A05206

29 Chen L, and Hasegawa A. A theory of long period magnetic pulsation 1: Steady state excitation of field line resonance. J Geophys Res, 1974, 79: $1024-1032$

30 Goertz C K, Boswell R W. Magnetosphere-ionosphere coupling. J Geophys Res, 1979, 84: 7239-7246

31 Chen L. Parametric excitation of 'kinetic' Alfvén waves by whistler waves. Plasma Phys, 1977, 19: 47-51

32 Papadopoulos K, Sharma R R, Tripathi V K. Parametric excitation of Alfvén waves in the ionosphere. J Geophys Res, 1982, 87: 14911494

33 Fedun V N, Yukhimuk A K, Voitsekhovskaya A D. The transformation of MHD Alfvén waves in space plasma. J Plasma Phys, 2004, 70: 699707

34 Zhao J S, Wu D J, Lu J Y. On nonlinear decay of kinetic Alfvén waves and application to some processes in space plasmas. J Geophys Res, 2010, 115: A12227

35 Brodin G, Stenflo L, Shukla P K. Nonlinear interactions between kinetic Alfvén and ion-sound waves. Solar Phys, 2006, 236: 285-291

36 Erokhin, N S, Moiseev S S, Mukhin V V. Decay instability of Alfvén waves in a hot plasma. Sov J Plasma Phys, 1978, 4: 656-657

37 Volokitin A S, Dubinin E M. The turbulence of Alfvén waves in the polar magnetosphere of the earth. Planet Space Sci, 1989, 37: 761-765

38 Frycz P, Rankin R, Samson J C, et al. Nonlinear field line resonances: Dispersive effects. Phys Plasmas, 1998, 5: 3565-3574

39 Samson J C, Rankin R, Tikhonchuk V T. Optical signatures of auro- ral arcs produced by field line resonances: Comparison with satellite observations and modeling. Ann Geophys, 2003, 21: 933-945

40 Rankin R, Lu J Y, Marchand R, et al. Spatiotemporal characteristics of ultra-low frequency dispersive scale shear Alfvén waves in the Earth's magnetosphere. Phys Plasmas, 2004, 11: 1268-1276

41 Rankin R, Kabin K, Lu J Y, et al. Magnetospheric field-line resonances: Ground-based observations and modeling. J Geophys Res, 2005, 110 A10S09

42 Rankin R, Marchand R, Lu J Y, et al. Theory of dispersive shear Alfvén wave focusing in Earth's magnetosphere. Geophys Res Lett, 2005, 32: L05102

43 Lundin R, Eliasson L, Haerendel G, et al. Large-scale auroral plasma density cavities observed by Freja. Geophys Res Lett, 1994, 21: 1903 1906

44 Stasiewicz K, Gustafsson G, Marklund G, et al. Cavity resonators and Alfvén resonance cones observed on Freja. J Geophys Res, 1997, 102: 2565-2575

45 Lu J Y, Rankin R, Marchand R, et al. Nonlinear electron heating by resonant shear Alfvén waves in the ionosphere. Geophys Res Lett, 2005 , 32: L01106

46 Lu J Y, Rankin R, Marchand R. Reply to comment by J.-P. St.-Maurice on "Nonlinear electron heating by resonant shear Alfvén waves in the ionosphere". Geophys Res Lett, 2005, 32: L13103

47 Noel J-M A, St-Maurice J P, Blelly P L. Nonlinear model of short-scale electrodynamics in the auroral ionosphere. Ann Geophys, 2000, 18: 1128-1144

48 Lanchester B S, Rees M H, Lummerzheim D, et al. Ohmic heating as evidence for strong field-aligned currents in filamentary aurora. J Geophys Res, 2001, 106: 1785-1794

49 Prakash M, Rankin R, Tikhonchuk V T. Precipitation and nonlinear effects in geomagnetic field line resonances. J Geophys Res, 2003, 108: 8014

50 Fedder J A, Slinker S P, Lyon J G, et al. Global numerical simulation of the growth phase and the expansion onset for a substorm observed by viking. J Geophys Res, 1995, 100: 19083-19093

51 Slinker S, Fedder J, Emery B, et al. Comparison of global MHD simulations with AMIE simulations for the events of May 19-20, 1996. J Geophys Res, 1999, 104: 28379-28395

52 Chiu Y T, Cornwall J M. Electrostatic model of a quiet auroral arc. J Geophys Res, 1980, 85: 543-556

53 Chiu Y T, Newman A L, Cornwall J M. On the structures and mapping of auroral electrostatic potentials. J Geophys Res, 1981, 86: 1002910037

54 Newell P T, Meng C-I, Wing S. Relation to solar activity of intense aurorae in sunlight and darkness. Nature, 1998, 393: 342-344

55 Liou K, Newell P T, Meng C-I. Seasonal effects on auroral particle acceleration and precipitation. J Geophys Res, 2001, 106: 5531-5542

56 Kletzing C A, Torbert R B. Electron time dispersion. J Geophys Res, 1994, 99: 2159-2172

57 Kletzing C A, Mozer F S, Torbert R B. Electron temperature and density at high latitude. J Geophys Res, 1998, 103: 14837-14845

58 Lysak R L, Lotko W. On the kinetic dispersion relation for shear Alfvén waves. J Geophys Res, 1996, 101: 5085-5094

59 Chaston C C, Carlson C W, Peria W J, et al. FAST observations of inertial Alfvén waves in the dayside aurora. Geophys Res Lett, 1999, 26 : 647-650

60 Lysak R L, Carlson C W. The effect of microscopic turbulence on magnetosphere-ionosphere coupling. Geophys Res Lett, 1981, 8: 269272

61 Andersson L, Ergun R E. Acceleration of antiearthward electron fluxes in the auroral region. J Geophys Res, 2006, 111: A07203

62 Zhao J S, Wu D J, Lu J Y. A nonlocal wave-wave interaction among Alfvén waves in an intermediate- $\beta$ plasma. Phys Plasmas, 2011, 18: 032903

Open Access This article is distributed under the terms of the Creative Commons Attribution License which permits any use, distribution, and reproduction in any medium, provided the original author(s) and source are credited. 